\title{
A multidisciplinary approach to complex oncological spine coverage in high-risk patients
}

\author{
Minh H. Nguyen, MD, ${ }^{1}$ Krishna Patel, MD, ${ }^{1}$ Julie West, MS, PA-C, ${ }^{1}$ Thomas Scharschmidt, MD, ${ }^{3}$ \\ Matthew Chetta, MD, ${ }^{1}$ Steven Schulz, MD, ${ }^{1}$ Ehud Mendel, MD, MBA, ${ }^{2}$ \\ and lan L. Valerio, MD, MS, MBA ${ }^{4}$
}

Departments of ${ }^{1}$ Plastic and Reconstructive Surgery, ${ }^{2}$ Neurosurgery, and ${ }^{3}$ Orthopedic Surgery, The Ohio State Wexner Medical Center, Columbus, Ohio; and ${ }^{4}$ Division of Plastic and Reconstructive Surgery, Massachusetts General Hospital, Harvard Medical School, Boston, Massachusetts

\begin{abstract}
OBJECTIVE The consequences of failed spinal hardware secondary to wound complications can increase the burden on the patient while also significantly escalating the cost of care. The objective of this study was to demonstrate the effectiveness of a protocol-based multidisciplinary approach in optimizing wound outcome in complex oncological spine care patients.
\end{abstract}

METHODS A retrospective consecutive case series was performed from 2015 to 2019 of all patients who underwent oncological spine surgery. A protocol was established to identify oncological patients at high risk for potential wound complications. Preoperative and postoperative treatment plans were developed by the multidisciplinary tumor board team members. Wound healing risk factors such as diabetes, obesity, prior spine surgery, pre- or postoperative chemotherapy or radiation exposure, perioperative steroid use, and poor nutritional status were recorded. Operative details, including the regions of spinal involvement, presence of instrumentation, and number of vertebral levels operated on, were reviewed. Primary outcomes were the length of hospitalization and major (requiring reoperation) and minor wound complications in the setting of the aforementioned identified risk factors.

RESULTS A total of 102 oncological cases were recorded during the 5-year study period. Of these patients, 99 had local muscle flap reconstruction with layered closure over their surgical hardware. The prevalence of smoking, diabetes, and previous spine surgery for the cohort was $21.6 \%, 20.6 \%$, and $27.5 \%$, respectively. Radiation exposure was seen in $72.5 \%$ of patients (37.3\% preoperative vs $48 \%$ postoperative exposure). Chemotherapy was given to $66.7 \%$ of the patients ( $49 \%$ preoperatively and $30.4 \%$ postoperatively). The rate of perioperative steroid exposure was $60.8 \%$. Prealbu$\mathrm{min}$ and albumin levels were $15.00 \pm 7.47 \mathrm{mg} / \mathrm{dL}$ and $3.23 \pm 0.43 \mathrm{mg} / \mathrm{dL}$, respectively. Overall, an albumin level of $<3.5$ $\mathrm{mg} / \mathrm{dL}$ and $\mathrm{BMl}<18.5$ were seen in $64.7 \%$ and $13.7 \%$ of the patients, respectively. The mean number of vertebral levels involved was $6.76 \pm 2.37$ levels. Instrumentation of 7 levels or more was seen in $52.9 \%$ of patients. The average spinal wound defect size was $22.06 \pm 7.79 \mathrm{~cm}$. The rate of minor wound complications, including superficial skin breakdown (epidermolysis) and nonoperative seromas, was $6.9 \%$, whereas that for major complications requiring reoperation within 90 days of surgery was $2.9 \%$.

CONCLUSIONS A multidisciplinary team approach utilized in complex multilevel oncological spine reconstruction surgery optimizes surgical outcomes, reduces morbidities, and improves care and satisfaction in patients with known risk factors.

https://thejns.org/doi/abs/10.3171/2020.6.SPINE2024

KEYWORDS oncological spine surgery; multidisciplinary; protocol; oncology

$\mathrm{S}$ URGICAL closure following oncological spine surgery is both complex and challenging. Surgical teams are faced with a patient population that has a high incidence of perioperative exposure to both pre- and postoperative chemotherapy and radiation therapy, malnutrition, and steroid therapy. Normal healing after complex onco- logical spinal surgery is attenuated, and the current literature reports major wound complications requiring reoperation in approximately $3.3 \%-32 \%$ of patients. ${ }^{1-3}$ Known surgical site complications such as seroma, hematoma, infection, wound breakdown, and radiation-induced microvascular injury are especially common in oncological 
spine surgery patients. Additionally, the high prevalence of smoking, diabetes, obesity, neurovascular compromise, chronic inflammation, and trauma creates a nonideal setting for wound healing in such patients when present. ${ }^{4}$ The consequences of failed spinal hardware include increased length of hospital stay, return to the operating room, readmission during the 30- to 90-day global health period, and economic and emotional burden to the healthcare system and patient. ${ }^{5-7}$

Recently, increased attention has been directed toward the construction and utilization of evidence-based protocols for risk reduction in various surgical patient cohorts. The implementation of such protocols has demonstrated a reduction in such prevalent postoperative complications, including thromboembolic events, surgical and drug-resistant bacterial infections, sepsis, and other related morbidities. ${ }^{8,9}$ The adoption of protocol-based care has been shown to positively impact resource utilization while decreasing costs via improvement in operational efficiencies and care rendered during surgical hospitalizations. ${ }^{9,10}$

Here, we report a consecutive retrospective study looking at the effectiveness of a multidisciplinary approach for postsurgical complication reduction (seroma, hematoma, wound infection, and wound dehiscence) in patients undergoing complex oncological spine reconstruction.

\section{Methods}

After obtaining appropriate IRB approval from The Ohio State University Wexner Medical Center, a retrospective consecutive case series study was performed on patients who underwent complex oncological spine reconstruction via a multidisciplinary approach at our institution between 2015 and 2019. The protocol used at our institution to guide treatment in these patients is demonstrated in Fig. 1. Our protocol encouraged early multidisciplinary involvement by the neurosurgery, orthopedic oncology, plastic surgery, surgical, medical, and radiation oncology teams in all patients who required $>3$ levels of instrumentation, perioperative exposure to steroids, chemotherapy or radiation therapy, a history of previous spine surgery, and multiple risk factors for poor wound healing, as determined by the preoperative assessment. This process was started during the weekly multidisciplinary tumor board meeting. During this meeting, patients having the previously described risk factor profile were identified and thus triaged into individualized treatment plans according to protocols designed by the team. Imaging, including CT scans, MR images, and PET scans, were collectively reviewed by the surgical teams to determine the resection parameters, the anticipated post-extirpative defect and tissues involved, and assessment of the local muscles that were anticipated to remain after oncological extirpation for flap reconstruction (i.e., assessment of the paraspinous, trapezius, and/or latissimus dorsi muscle flaps).

Perioperative optimization of the patients was also pursued. Specifically, cachectic patients or those with prealbumin $<16 \mathrm{mg} / \mathrm{dL}$ or albumin $<3.5 \mathrm{mg} / \mathrm{dL}$ were provided with oral protein supplements. ${ }^{11}$ Vitamin A and micronutrient supplements (zinc and selenium) were provided to patients with perioperative steroid exposure. Patients with prior radiation therapy were placed on a micronutrient supplement. If patients were deemed to have poor skin quality or multiple risk factors for poor wound healing (e.g., malnutrition, perioperative steroid exposure, prior radiation exposure), negative pressure wound therapy was considered as an adjunct to their surgical flap coverage procedure to help optimize their wound healing. If the spinal defect was not appropriate for flap coverage, the plastic surgery team was on standby for intraoperative consultation if needed by the primary team. For those patients who underwent urgent surgeries due to unexpected events such as pathological fracture with acute spinal cord compression secondary to diseases, similar protocols were implemented as soon as the patient was admitted to the hospital.

In patients who fit our multidisciplinary protocol, comorbid conditions, including the prevalence of diabetes, smoking, previous spine surgery, and obesity, were reviewed. Risk factors leading to impaired wound healing (e.g., the presence of pre- or postoperative chemotherapy or radiation therapy, perioperative steroid use, and poor nutritional status [measured via prealbumin and albumin levels]) were also recorded. Details regarding the spine surgeries including the spine region (cervical, thoracic, lumbar, sacral spine, and/or pelvis), presence of instrumentation, and number of spinal levels operated on were also tabulated. The primary outcomes that were measured included the length of hospitalization and major and minor wound complications. Major complications were defined as those requiring a return to the operating room within 90 days, while minor complications were those that were managed on an outpatient basis (e.g., superficial wound dehiscence, nonoperative seromas, hematomas, or surgical site infections [SSIs; including cellulitis and drain site cellulitis]).

\section{Results}

A total of 102 multidisciplinary oncological spine reconstructions were identified between 2015 and 2019. The average age at surgery was $59.61 \pm 12.98$ years $( \pm$ SD), with 50 females and 52 males. Comorbid conditions included smoking in $21.6 \%$, diabetes in $20.6 \%$, and previous spine surgery in $27.5 \%$ of patients. Of the 102 patients, radiation exposure was seen in $72.5 \%$ (37.3\% preoperatively and $48 \%$ postoperatively). Chemotherapy was given to $66.7 \%$ of the patients (49\% preoperatively and $30.4 \%$ postoperatively). Perioperative steroid exposure was seen in $60.8 \%$ of patients. Perioperative nutritional status was measured by prealbumin and albumin levels, which were $15.00 \pm$ $7.47 \mathrm{mg} / \mathrm{dL}$ and $3.23 \pm 0.43 \mathrm{mg} / \mathrm{dL}$, respectively. Malnutrition, defined as albumin $<3.5 \mathrm{mg} / \mathrm{dL}$, was seen in $64.7 \%$ of the patients; $13.7 \%$ of the patients also had a BMI $<18.5$. Patient demographics and risk factors are summarized in Table 1.

As a proxy measurement for surgical complexity, the mean number of vertebral levels involved and size of the wound defect were $6.76 \pm 2.37$ levels $(52.9 \%$ with instrumentation of 7 levels or more) and $22.06 \pm 7.79 \mathrm{~cm}$ (range 6-60 $\mathrm{cm}$ ), respectively. Spinal surgery involved single, double, or triple regions in $48 \%, 41.2 \%$, and $10.8 \%$ of cases, respectively. En bloc resections for spinal tumors were 


\section{Criteria for Early Multidisciplinary Involvement*}

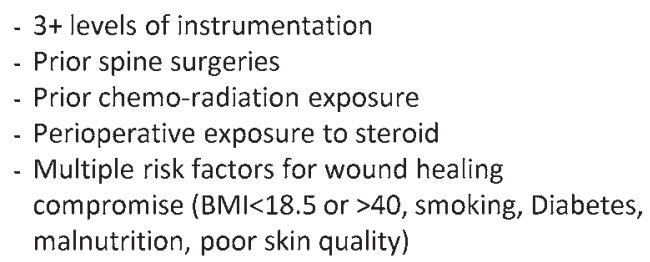

\section{Perioperative Optimization}

- If Pre-albumin $<16$ or Albumin $<3.5 \rightarrow$ Give
oral protein supplement. Consider tube feed if
patient cannot tolerate PO intake.
- If perioperative steroid is used $\rightarrow$ Give vitamin
A supplement and micronutrients (Zinc and
Selenium)
- If patient had prior radiation exposure $\rightarrow$ Give
micronutrients (Zinc and Selenium)
- If a patient has poor skin quality or one of the
above risk factors $\rightarrow$ Consider incisional
negative pressure wound therapy (NPWT). ${ }^{* *}$
Plastic surgery on stand-by for intraoperative consult if needed.

When applicable, perioperative optimization strategies with regards to nutrition, micronutrients and vitamin $\mathrm{A}$ supplementation were recommended.

FIG. 1. Multidisciplinary protocol for complex oncological spine coverage in high-risk patients. Patients with risk factors that fit the criteria were identified during the tumor board meeting. Perioperative optimization strategies were then initiated on appropriate patients. $\mathrm{PO}=$ by mouth. ${ }^{*}$ This decision is made during the weekly tumor board meeting. ${ }^{* *}$ This decision can be made intraoperatively if the surgery is urgent.

achieved in 10 cases $(9.8 \%)$. Operative details are summarized in Table 2.

Major wound complications that required surgery were seen in less than $3 \%$ of patients (3 of 102 cases). Minor wound complications were seen in $6.9 \%$ of patients $(7$ of 102 cases) and included nonoperative seroma, cellulitis, or superficial skin epidermolysis. The mean length of stay after surgery was $10.11 \pm 5.65$ days. Drains were kept in place for $22.15 \pm 12.57$ days on average. The mean length of follow-up was $293.5 \pm 373.69$ days. In terms of neurosurgical complications, CSF leakage occurred in 6 cases $(5.9 \%)$, and hardware failure was seen in 7 cases $(6.9 \%)$. Postoperative complications and follow-up details are summarized in Table 3.

\section{Discussion}

In our series of oncological spine patients, a high number of the patients were malnourished and were in a metabolic cachectic state on presentation. Additionally, many had other contributing factors that put them at a higher risk for postoperative complications. In general, this patient population has important risk factors that have demonstrated impedances to normal wound healing as well as increased rates of postoperative complications. ${ }^{12-17}$ Hyperglycemia seen in patients with poorly controlled diabetes is known to cause fibroblast and epidermal cell dysfunction, increased tissue hypoxia and oxidative dam- age from reactive oxygen species, and decreased angiogenesis and neovascularization, which ultimately result in impaired wound healing and host immune resistance to infections. ${ }^{18}$ In tobacco users, the vasoconstrictive effects of nicotine, coupled with cyanide-induced impaired cellular metabolism, have demonstrated impaired oxygen utilization in tissues, especially in healing wounds. ${ }^{19,20}$ Moreover, tobacco use results in impaired white blood cell and fibroblast migration as well as decreased neutrophilic bactericidal activity, thus predisposing the patients to poor wound healing and increased risk for opportunistic infections. ${ }^{19,21}$ Nutritionally depleted and protein-deficient patients have been shown to be at a higher risk of poor and delayed wound healing and increased rates of infection and dehiscence, and have high associated unplanned hospital readmission rates within 30 days of surgery. ${ }^{1722} \mathrm{Ra}-$ diation-induced fibrosis attenuates wound healing and increases rates of major and minor wound complications. ${ }^{17,23}$ Since most chemotherapeutic drugs are designed to inhibit cellular proliferation and angiogenesis, these drugs also inhibit pathways critical to adequate wound repair. ${ }^{24}$ Systemic steroids also inhibit wound repair via global immunosuppression and antiinflammatory effects, including decreased fibroblast proliferation and collagen synthesis. ${ }^{18}$

The high comorbidity risk profile in our cohort $(29.4 \%$ with obesity [BMI $\geq 30], 21.6 \%$ with active smoking status, $20.6 \%$ with diabetes, and $64.7 \%$ with albumin $<3.5$ $\mathrm{mg} / \mathrm{dL}$ ) coupled with high rates of pre- and postopera- 
TABLE 1. Patient demographics and risk factors $(n=102)$

\begin{tabular}{|c|c|}
\hline & Value \\
\hline Age in yrs, mean \pm SD & $59.61 \pm 12.98$ \\
\hline \multicolumn{2}{|l|}{ Sex, n (\%) } \\
\hline Male & $52(51)$ \\
\hline Female & $50(49)$ \\
\hline \multicolumn{2}{|l|}{ Comorbidities, n (\%) } \\
\hline Diabetes mellitus & $21(20.6)$ \\
\hline Obesity & $30(29.4)$ \\
\hline BMI 30-34.9 & $17(16.7)$ \\
\hline BMI 35-39.9 & $7(6.9)$ \\
\hline $\mathrm{BMI}>40$ & $6(5.9)$ \\
\hline Current smoker & $22(21.6)$ \\
\hline \multicolumn{2}{|l|}{ Malnutrition } \\
\hline Albumin $<3.5 \mathrm{mg} / \mathrm{dL}$ & $66(64.7)$ \\
\hline $\mathrm{BMI}<18.5$ & $14(13.7)$ \\
\hline Previous spine surgery, $\mathrm{n}(\%)$ & $28(27.5)$ \\
\hline \multicolumn{2}{|l|}{ Surgical indication, $\mathrm{n}(\%)$} \\
\hline Primary cancer & $23(22.5)$ \\
\hline Metastatic cancer & $79(77.5)$ \\
\hline \multicolumn{2}{|c|}{ Pre- \& postop treatment exposure, $n(\%)$} \\
\hline Chemotherapy & $68(66.7)$ \\
\hline Preop & $50(49.0)$ \\
\hline Postop & $31(30.4)$ \\
\hline Radiation therapy & $74(72.5)$ \\
\hline Preop & $38(37.3)$ \\
\hline Postop & $49(48.0)$ \\
\hline Steroids & $62(60.8)$ \\
\hline
\end{tabular}

tive exposure to radiation therapy $(72.5 \%)$, chemotherapy (66.7\%), and steroids (60.8\%) are critical issues to consider and have been reported as independent risk factors for wound healing delay with associated elevated complication rates. ${ }^{6,8,14,16,24}$ As also seen within this series, oncological spine patients often require multiple levels of instrumentation for spinal stability. A recent systematic review of 262 patients undergoing flap-based spinal reconstruction proposed that prophylactic flap coverage in spinal patients with multilevel instrumentation reduced the incidence of postoperative wound complications. ${ }^{25}$ Additionally, other publications have shown that spinal reconstruction surgeries involving 7 or more levels of stabilization hardware had significantly increased rates of SSI and wound healing problems ${ }^{15}$ Since perioperative oncological treatment and instrumentation placement is often necessary given the oncological disease burden, prophylactic coverage with durable and vascularized muscle flaps is imperative in improving surgical outcomes and reducing wound complications in these patients.

The diminished healing capacity in patients undergoing oncological spinal reconstruction confers increased predisposition to common surgical complications such as SSIs, hematomas, seromas, and dehiscence. ${ }^{14,26,27}$ In their meta-analysis, Bernatz and Anderson reported SSIs as be-
TABLE 2. Operative details

\begin{tabular}{cc}
\hline & Value \\
\hline Incision length in cm, mean \pm SD & $22.06 \pm 7.79$ \\
\hline En bloc resection, $\mathrm{n}(\%)$ & $10(9.8)$ \\
\hline Surgical levels, $\mathrm{n}(\%)$ & $3(2.9)$ \\
\hline Cervical & $43(42.2)$ \\
\hline Thoracic & $0(0)$ \\
\hline Lumbar & $3(2.9)$ \\
\hline Sacral & $17(16.7)$ \\
\hline Cervical, thoracic & $18(17.6)$ \\
\hline Thoracic, lumbar & $7(6.9)$ \\
\hline Lumbar, sacral & $11(10.8)$ \\
\hline Lumbar, sacral, pelvis & $0(0)$ \\
\hline Thoracic, lumbar, sacral, pelvic & $88(86.3)$ \\
\hline Instrumentation placed, $\mathrm{n}(\%)$ & $6.76 \pm 2.37$ \\
\hline No. of instrumented levels, mean \pm SD & $54(52.9)$ \\
\hline$\geq 7$ levels, $\mathrm{n}$ (\%) & $99(97.1)$ \\
\hline Reconstruction, $\mathrm{n}(\%)$ & \\
\hline Muscle flap coverage & $96(94.1)$ \\
\hline Bilat paraspinous & $39(38.2)$ \\
\hline Bilat trapezius & $37(36.3)$ \\
\hline Bilat paraspinous + bilat trapezius & $3(2.9)$ \\
\hline Latissimus dorsi (uni- \& bilat) & $7(6.9)$ \\
\hline Gluteal fasciocutaneous advancement & $3(2.9)$ \\
\hline flap (uni- \& bilat) &
\end{tabular}

ing the most common cause of readmission within 30 days after spinal surgery. ${ }^{29}$ The estimated cost of treating these SSIs ranges from $\$ 12,600$ to $\$ 38,000$ for adults and can result in a $60 \%$ increase in the mean cost of stay, with over one-third of this due to the need of additional procedures. ${ }^{28-31}$ Not only are postsurgical complications expensive, they are also associated with an increased length of hospital stay resulting in exposure to pneumonia, deep vein thrombosis, pulmonary emboli, pressure ulcers, and urinary tract infections. ${ }^{32-34}$ Furthermore, major complications requiring a return to the operating room are associated with increased technical difficulty and can potentially increase infection transposition into the CNS, resulting in chronic pain, paralysis, deformity, or even death. . $27,35,36^{2}$ Complications requiring reoperation also increase emotional and economic burden, decrease quality of life, and cause unnecessary delay in adjuvant chemotherapy or radiation therapy in oncology patients. ${ }^{14,29}$ Thus, avoidance of complications should play a greater than normal role in the surgical decision-making, as complications in these high-risk patients is associated with significant morbidity and extensive resource utilization.

Flap reconstruction to cover spine hardware alone is not sufficient to optimize surgical outcomes in the oncological spine patient. Adoption and implementation of appropriate protocol-based practice guidelines have demonstrated improved hospital resource allocation and reduced 
TABLE 3. Postoperative details and complications

\begin{tabular}{cc}
\hline & Value \\
\hline Length of hospital stay in days, mean \pm SD & $14.68 \pm 8.08$ \\
\hline After surgery & $10.11 \pm 5.65$ \\
\hline Time before drain removal in days, mean \pm SD & $22.15 \pm 12.57$ \\
\hline Major wound complications, $\mathrm{n}(\%)$ & $3(2.9)$ \\
\hline Hematoma* & $1(1.0)$ \\
\hline Major wound infection* & $1(1.0)$ \\
\hline Major wound dehiscence* & $2(2.0)$ \\
\hline Seroma requiring op & $1(1.0)$ \\
\hline Minor wound complications, $\mathrm{n}(\%)$ & $7(6.9)$ \\
\hline Seroma requiring no op & $1(1.0)$ \\
\hline Superficial skin breakdown (epidermolysis) & $6(5.9)$ \\
\hline Length of follow-up in days, mean \pm SD & $293.5 \pm 373.69$ \\
\hline Spine surgery-related complications, $\mathrm{n}(\%)$ & $16(15.7)$ \\
\hline CSF leak & $6(5.9)$ \\
\hline Hardware failure requiring revision & $7(6.9)$ \\
\hline Other & $3(2.9)$ \\
\hline 30-day mortality, $\mathrm{n}(\%) \dagger$ & $4(3.9)$ \\
\hline
\end{tabular}

* One patient had operative wound dehiscence and hematoma, and another patient had operative wound dehiscence and infection.

$\dagger$ All related to disease burden.

adverse effects. ${ }^{8-10}$ Incorporating multidisciplinary care teams further improves surgical outcomes, reduces complications, and improves patient satisfaction. The ability and expertise of the reconstructive surgeons to adequately cover large, critical defects with local autologous flaps, negative pressure wound therapy, acellular dermal matrices, and staging operations has resulted in the reduction of postsurgical infections and unplanned reoperations, while demonstrating improved functionality. ${ }^{23}$ This has been reliably demonstrated and reported in various areas of plastic and reconstructive surgery. A neuroplastic approach for the surgical management of myelomeningoceles has yielded significantly reduced rates of CSF leaks, infection, and surgical site dehiscence. ${ }^{7}$ An oncoplastic-based chest wall reconstruction protocol has led to improved functional and cosmetic outcomes, with lower infection rates following malignant chest wall tumor resections. ${ }^{37}$ It has also been shown that combined ortho-onco-plastic care of amputee patients with concurrent targeted muscle reinnervation during amputations has led to a reduced incidence and severity of neuromas and phantom limb pain. ${ }^{22}$ These protocols have demonstrated improved surgical outcomes with lower rates of complications and have supported the use of multidisciplinary care teams to augment patient care. Other reports have corroborated these findings and emphasized the use of immediate flap-based reconstruction in patients with larger defects and those with extensive risk factors for poor wound healing. ${ }^{24}$ With low rates of major (2.9\%) and minor (6.9\%) complications in our patients, compared with the $3.3 \%-32 \%$ postsurgical complications seen in the literature, our study encourages the use of a multidisciplinary protocol to improve surgical outcomes when taking a variety of preoperative risk fac- tors into consideration and implementing care plans to aid in risk reduction.

The limitations of our study include its retrospective study design and the lack of a morbidity-matched control group of spinal surgery patients managed without multidisciplinary care intervention. However, published data in the current literature helped validate the result of our approach. In a recent publication by Leary et al., which compared the neuroplastic approach with primary closure by neurosurgery, the neuroplastic approach group had similar complication rates despite the greater usage of instrumentation, number of levels instrumented, and pre- or postoperative radiation exposure..$^{38}$ Our study also evaluated similar risk factors similar to those mentioned by Leary et al. The risk factors in the neuroplastic group included BMI $28.1 \pm 5.4$, smoking $(38 / 60,58 \%)$, diabetes $(5 / 60$, $8 \%$ ), preoperative radiation exposure $(32 / 60,53 \%)$, postoperative radiation exposure $(31 / 60,52 \%)$, preoperative chemotherapy $(22 / 60,37 \%)$, postoperative chemotherapy $(19 / 60,32 \%)$, mean number of instrumented levels $(5.1 \pm$ 3.8 levels), mean wound length $(22.6 \pm 7.1 \mathrm{~cm})$, and instrumentation usage $(45 / 60,75 \%)$. Their rate of major wound complications requiring surgery was $17 \%$ (10/60) while their total wound complication rate was $28 \%$ (17/60). Nevertheless, this comparison does not replace the need for a good prospective randomized controlled trial in potential future studies to demonstrate the risk reduction with the use of evidence-based protocols. Looking forward, subsequent studies can differentiate outcomes based on length and/or total dosage of chemoradiation and steroid exposure, subtypes of neoplasm resected, genetic factors that may influence wound healing or tumor response, and the levels/regions of spinal instrumentation to help minimize the sample population heterogeneity.

\section{Conclusions}

A multidisciplinary team approach utilized in complex multilevel oncological spine reconstruction surgery optimizes surgical outcomes, reduces morbidity, and improves care and satisfaction in patients with known risk factors.

\section{References}

1. Chang DW, Friel MT, Youssef AA. Reconstructive strategies in soft tissue reconstruction after resection of spinal neoplasms. Spine (Phila Pa 1976). 2007;32(10):1101-1106.

2. Franck P, Bernstein JL, Cohen LE, et al. Local muscle flaps minimize post-operative wound morbidity in patients with neoplastic disease of the spine. Clin Neurol Neurosurg. 2018; 171:100-105.

3. Garvey PB, Rhines LD, Dong W, Chang DW. Immediate soft-tissue reconstruction for complex defects of the spine following surgery for spinal neoplasms. Plast Reconstr Surg. 2010;125(5):1460-1466.

4. Janis JE, Harrison B. Wound healing: part I. Basic science. Plast Reconstr Surg. 2016;138(3)(suppl):9S-17S.

5. Lau D, Chan AK, Theologis AA, et al. Costs and readmission rates for the resection of primary and metastatic spinal tumors: a comparative analysis of 181 patients. J Neurosurg Spine. 2016;25(3):366-378.

6. Mericli AF, Rhines L, Bird J, et al. Immediate reconstruction of oncologic spinal wounds is cost-effective compared with 
conventional primary wound closure. Plast Reconstr Surg. 2019;144(5):1182-1195.

7. Parker SL, Shau DN, Mendenhall SK, McGirt MJ. Factors influencing 2-year health care costs in patients undergoing revision lumbar fusion procedures. J Neurosurg Spine. 2012; 16(4):323-328.

8. González Della Valle A, Serota A, Go G, et al. Venous thromboembolism is rare with a multimodal prophylaxis protocol after total hip arthroplasty. Clin Orthop Relat Res. 2006;444(444):146-153.

9. Talmor D, Greenberg D, Howell MD, et al. The costs and cost-effectiveness of an integrated sepsis treatment protocol. Crit Care Med. 2008;36(4):1168-1174.

10. Halpin RJ, Sugrue PA, Gould RW, et al. Standardizing care for high-risk patients in spine surgery: the Northwestern high-risk spine protocol. Spine (Phila Pa 1976). 2010;35(25): 2232-2238.

11. Riaz O, Arshad R, Nisar S, Vanker R. Serum albumin and fixation failure with cannulated hip screws in undisplaced intracapsular femoral neck fracture. Ann R Coll Surg Engl. 2016;98(6):376-379.

12. Anderson PA, Savage JW, Vaccaro AR, et al. Prevention of surgical site infection in spine surgery. Neurosurgery. 2017; 80(3S):S114-S123.

13. Bohl DD, Shen MR, Mayo BC, et al. Malnutrition predicts infectious and wound complications following posterior lumbar spinal fusion. Spine (Phila Pa 1976). 2016;41(21): 1693-1699.

14. Dolan RT, Butler JS, Wilson-MacDonald J, et al. Quality of life and surgical outcomes after soft-tissue reconstruction of complex oncologic defects of the spine and sacrum. J Bone Joint Surg Am. 2016;98(2):117-126.

15. Kumar S, van Popta D, Rodrigues-Pinto R, et al. Risk factors for wound infection in surgery for spinal metastasis. Eur Spine J. 2015;24(3):528-532.

16. McMurry JF Jr. Wound healing with diabetes mellitus. Better glucose control for better wound healing in diabetes. Surg Clin North Am. 1984;64(4):769-778.

17. Wise JJ, Fischgrund JS, Herkowitz HN, et al. Complication, survival rates, and risk factors of surgery for metastatic disease of the spine. Spine (Phila Pa 1976). 1999;24(18): 1943-1951.

18. Guo S, Dipietro LA. Factors affecting wound healing. J Dent Res. 2010;89(3):219-229.

19. Ahn C, Mulligan P, Salcido RS. Smoking - the bane of wound healing: biomedical interventions and social influences. Adv Skin Wound Care. 2008;21(5):227-236.

20. Sørensen LT, Jørgensen S, Petersen LJ, et al. Acute effects of nicotine and smoking on blood flow, tissue oxygen, and aerobe metabolism of the skin and subcutis. J Surg Res. 2009; 152(2):224-230.

21. McMaster SK, Paul-Clark MJ, Walters M, et al. Cigarette smoke inhibits macrophage sensing of Gram-negative bacteria and lipopolysaccharide: relative roles of nicotine and oxidant stress. Br J Pharmacol. 2008;153(3):536-543.

22. Alexander JH, Jordan SW, West JM, et al. Targeted muscle reinnervation in oncologic amputees: early experience of a novel institutional protocol. J Surg Oncol. 2019;120(3): 348-358.

23. Devulapalli C, Broyles JM, Bello R, et al. Soft-tissue reconstruction of large spinal defects: a 12-year institutional experience. Plast Reconstr Surg. 2017;140(4):806-814.

24. Franz MG, Steed DL, Robson MC. Optimizing healing of the acute wound by minimizing complications. Curr Probl Surg. 2007:44(11):691-763

25. Chieng LO, Hubbard Z, Salgado CJ, et al. Reconstruction of open wounds as a complication of spinal surgery with flaps: a systematic review. Neurosurg Focus. 2015;39(4):E17.

26. Mericli AF, Tarola NA, Moore JH, et al. Paraspinous muscle flap reconstruction of complex midline back wounds: risk factors and postreconstruction complications. Ann Plast Surg. 2010;65(2):219-224.

27. Omeis IA, Dhir M, Sciubba DM, et al. Postoperative surgical site infections in patients undergoing spinal tumor surgery: incidence and risk factors. Spine (Phila Pa 1976). 2011; 36(17):1410-1419.

28. Atkinson RA, Jones A, Ousey K, Stephenson J. Management and cost of surgical site infection in patients undergoing surgery for spinal metastasis. J Hosp Infect. 2017;95(2):148-153.

29. Bernatz JT, Anderson PA. Thirty-day readmission rates in spine surgery: systematic review and meta-analysis. Neurosurg Focus. 2015;39(4):E7.

30. Kuhns BD, Lubelski D, Alvin MD, et al. Cost and quality of life outcome analysis of postoperative infections after subaxial dorsal cervical fusions. J Neurosurg Spine. 2015;22(4): 381-386.

31. Yeramaneni S, Robinson C, Hostin R. Impact of spine surgery complications on costs associated with management of adult spinal deformity. Curr Rev Musculoskelet Med. 2016; 9(3):327-332.

32. Khalafallah AA, Kirkby BE, Wong S, et al. Venous thromboembolism in medical patients during hospitalisation and 3 months after hospitalisation: a prospective observational study. BMJ Open. 2016;6(8):e012346.

33. Lynch JP III. Hospital-acquired pneumonia: risk factors, microbiology, and treatment. Chest. 2001;119(2)(suppl):373S384 S.

34. Shah NK, Farber A, Kalish JA, et al. Occurrence of "never events" after major open vascular surgery procedures. J Vasc Surg. 2016;63(3):738-745.e28.

35. Calderone RR, Garland DE, Capen DA, Oster H. Cost of medical care for postoperative spinal infections. Orthop Clin North Am. 1996;27(1):171-182.

36. Zucherman J, Hsu K, White A, Wynne G. Early results of spinal fusion using variable spine plating system. Spine (Phila Pa 1976). 1988;13(5):570-579.

37. Khalil HH, Malahias MN, Balasubramanian B, et al. Multidisciplinary oncoplastic approach reduces infection in chest wall resection and reconstruction for malignant chest wall tumors. Plast Reconstr Surg Glob Open. 2016;4(7):e809.

38. Leary OP, Liu DD, Boyajian MK, et al. Complex wound closure by plastic surgery following resection of spinal neoplasms minimizes postoperative wound complications in high-risk patients. J Neurosurg Spine. 2020;33(1):77-86.

\section{Disclosures}

Dr. Scharschmidt: consultant for Stryker Orthopedics and Daiichi Sankyo. Dr. Valerio: consultant for Stryker, Axogen, and Integra LifeSciences.

\section{Author Contributions}

Conception and design: Valerio, Nguyen, West, Scharschmidt, Chetta, Schulz, Mendel. Acquisition of data: Nguyen, Patel, West. Analysis and interpretation of data: Valerio, Nguyen, West, Scharschmidt, Schulz, Mendel. Drafting the article: Nguyen, Patel. Critically revising the article: Valerio, Nguyen, West, Scharschmidt, Chetta, Schulz, Mendel. Reviewed submitted version of manuscript: all authors. Approved the final version of the manuscript on behalf of all authors: Valerio. Statistical analysis: Valerio, Nguyen. Administrative/technical/material support: West. Study supervision: Valerio, Schulz, Mendel.

\section{Correspondence}

Ian L. Valerio: Massachusetts General Hospital, Harvard Medical School, Boston, MA. ivalerio@mgh.harvard.edu. 